\title{
The design of an individualized cylindrical vaginal applicator with oblique guide holes using 3D modeling and printing technologies
}

\author{
Zhipeng Zhao, MS*, Xiaodi Tang, BA*, Zhuang Mao, BA, Hongfu Zhao, MS \\ Department of Radiation Oncology, China-Japan Union Hospital of Jilin University, Changchun, China \\ *Zhipeng Zhao and Xiaodi Tang contributed equally to this work.
}

\begin{abstract}
High-dose-rate brachytherapy for cervical cancer after subtotal hysterectomy using standardized applicators cannot achieve a good absorbed-dose coverage of the target volume in special tumor morphologies and topographies due to the steep dose gradient. The aim of this pictorial essay is to present an individualized cylindrical vaginal applicator with oblique guide holes using 3D modeling and printing technologies used at the China-Japan Union Hospital of Jilin University for cervical cancer patients. We use images to describe the steps of this method.
\end{abstract}

J Contemp Brachytherapy 2019; II, 5: 479-487 DOI: https://doi.org/10.5114/jcb.2019.88441

Key words: cervical cancer, brachytherapy, 3D modeling, 3D printing.

\section{Purpose}

External beam radiotherapy (EBRT), concomitant chemotherapy, and brachytherapy is the modern treatment modality for advanced cervical cancer. Brachytherapy is an integral part of radiation treatment of cervical cancer. Studies have shown a clear dose-volume effect between modern dosimetric parameters $\left(D_{90}\right.$ of high-risk clinical target volume [HR-CTV] and intermediate-risk clinical target volume [IR-CTV]) and the probability of achieving local control $[1,2]$. For cervical cancer brachytherapy, common standardized applicators include tandem and ring applicators, tandem and ovoid applicators, vaginal cylinders, combined intracavitary and interstitial (IC/IS) applicators, and the Martinez universal perineal interstitial template $[3,4,5,6]$. Each applicator has its own unique structural characteristics and is applicable to a specific situation.

For special tumor morphology and topography, brachytherapy cannot achieve a good absorbed-dose coverage of the target volume for using standardized applicators due to a steep dose gradient, thereby not achieving good local control. Many studies have made many attempts in the field of individualized applicators and have achieved good results $[7,8,9,10]$. The aim of this pictorial essay is to demonstrate the workflow of an individualized cylindrical vaginal applicator with oblique guide holes using 3D modeling and printing technologies.

\section{Material and methods}

A 47-year-old woman was diagnosed with stage IIIA cervical cancer after having a subtotal hysterectomy for uterine fibroids 10 years prior. A gynecological examination and magnetic resonance imaging (MRI) showed that the tumor was located at the anterior lip of the cervix, the upper one-third of the anterior wall of the vagina, and the left onethird of the parauterine organ, with a size of $5.6 \times 3 \times 3.8 \mathrm{~cm}^{3}$. The dose of EBRT was $45 \mathrm{~Gy} / 25$ fractions delivered by intensity-modulated radiation therapy. After EBRT, a gynecological examination showed that the tumor involved the upper one-third of the anterior vaginal wall with a $2 \mathrm{~cm}$ maximum extension in depth. In consideration of her stenotic vagina and tumor location, we applied the individualized vaginal cylinder applicator with guide holes. The design of the applicator was based on the preplanning steps: computed tomography (CT) simulation and contour (Figure 1), establishment of coordinate system of the applicator (Figure 2), optimized design of needle path (Figures 3-5), and dose distribution optimization (Figure 6). The delineation of the target volumes was contoured according to recommendations from the GYN GEC-ESTRO Working Group [11]. Data on the direction for the needles based on the coordinate system are shown in Table 1. According to the data from the preplanning phase, the 3D modeling of the applicator (Figures 7-10) was implemented in 3ds Max (2015 version, Autodesk Inc., San Rafael, CA, USA). The 3D printing process of the 

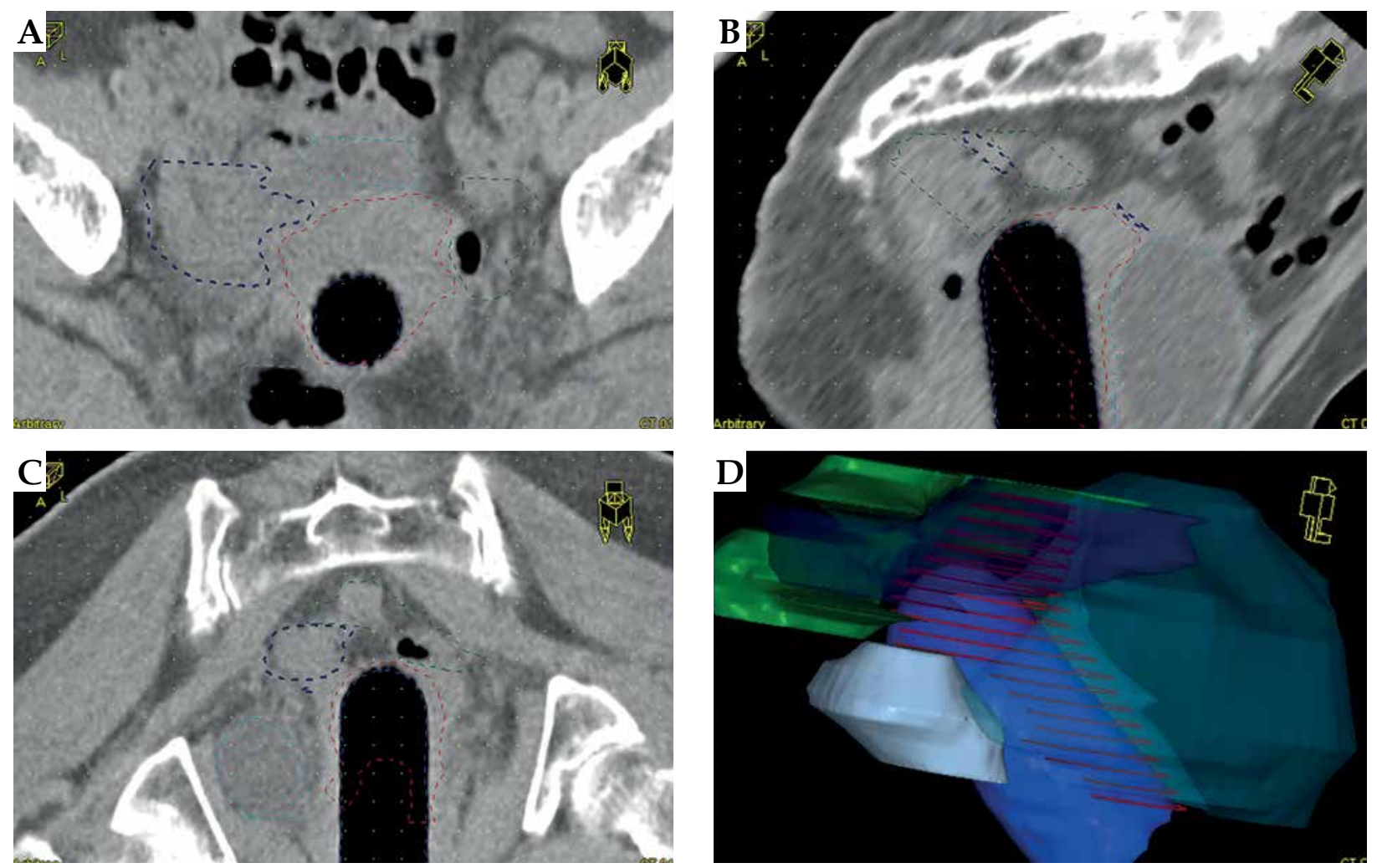

Fig. 1. I. Preplanning: CT simulation was performed after a vaginal model (a half sphere $3 \mathrm{~cm}$ in diameter and a cylinder $6 \mathrm{~cm}$ in length and $3 \mathrm{~cm}$ in diameter) was implanted into the vagina. The target volumes were delineated on reconstructed CT images with reference MR images before implantation. The red dotted line is the HR-CTV, and the green dotted line is the IR-CTV: A) para-axial plane (according to dummy applicator), B) sagittal plane, C) para-coronal plane (according to dummy applicator), D) 3D view

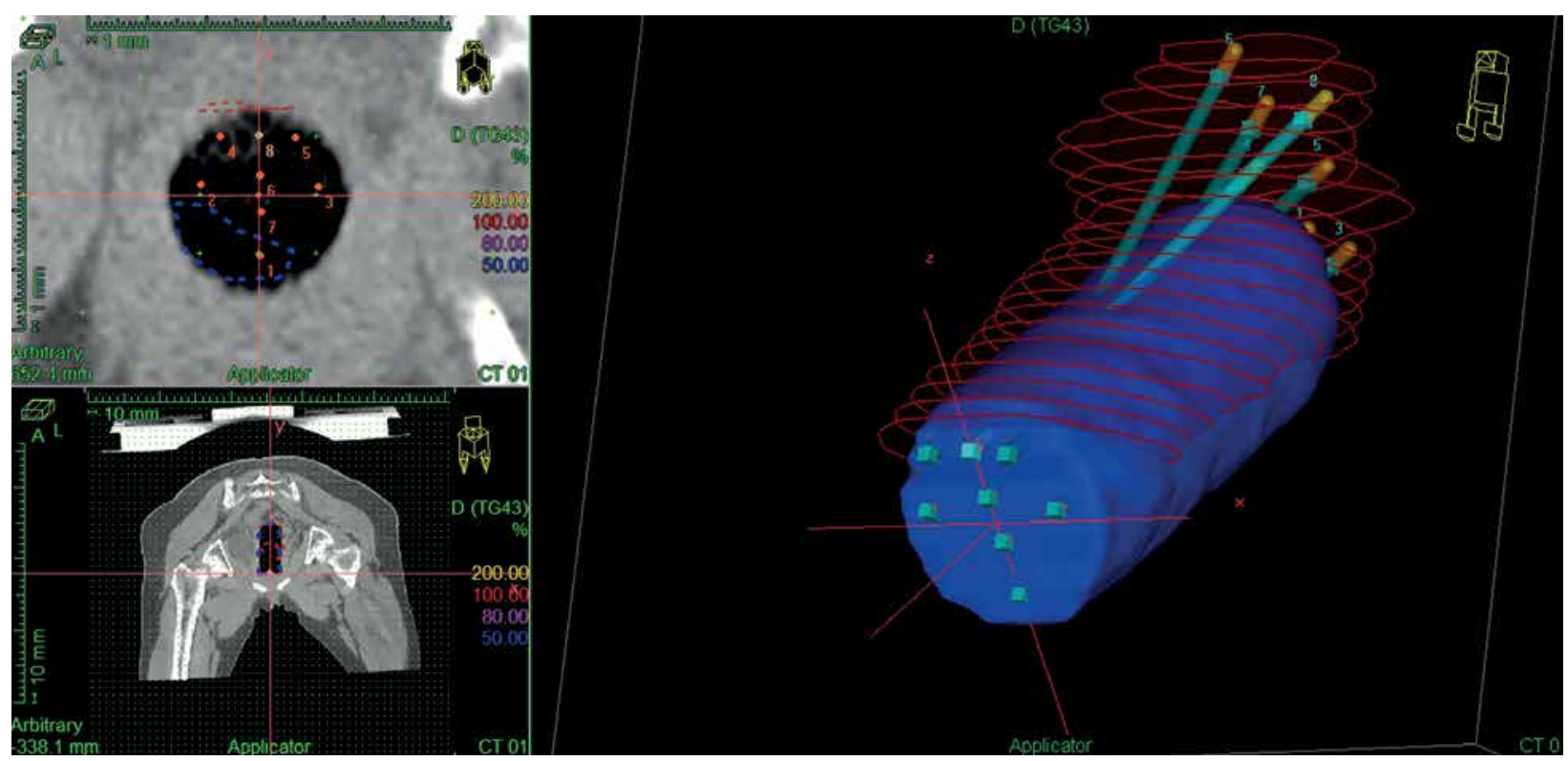

Fig. 2. I. Preplanning: To design convenient directions of the implanted needles, we established the coordinate system of the applicator as follows: the x-axis is the left and right direction, where the left of the patient is positive; the z-axis is the anterior and posterior direction, where the anterior of the patient is positive; the y-axis is the head and foot direction, and the head of the patient is positive. We designed an ideal implanted needle according to the relationship between the tumor (red coil) and the vaginal cylinder (blue) while considering the needle distribution. Two points determine a straight line (the implant needle). The superior point is called the "tip end", and the inferior point is called the "base end" 

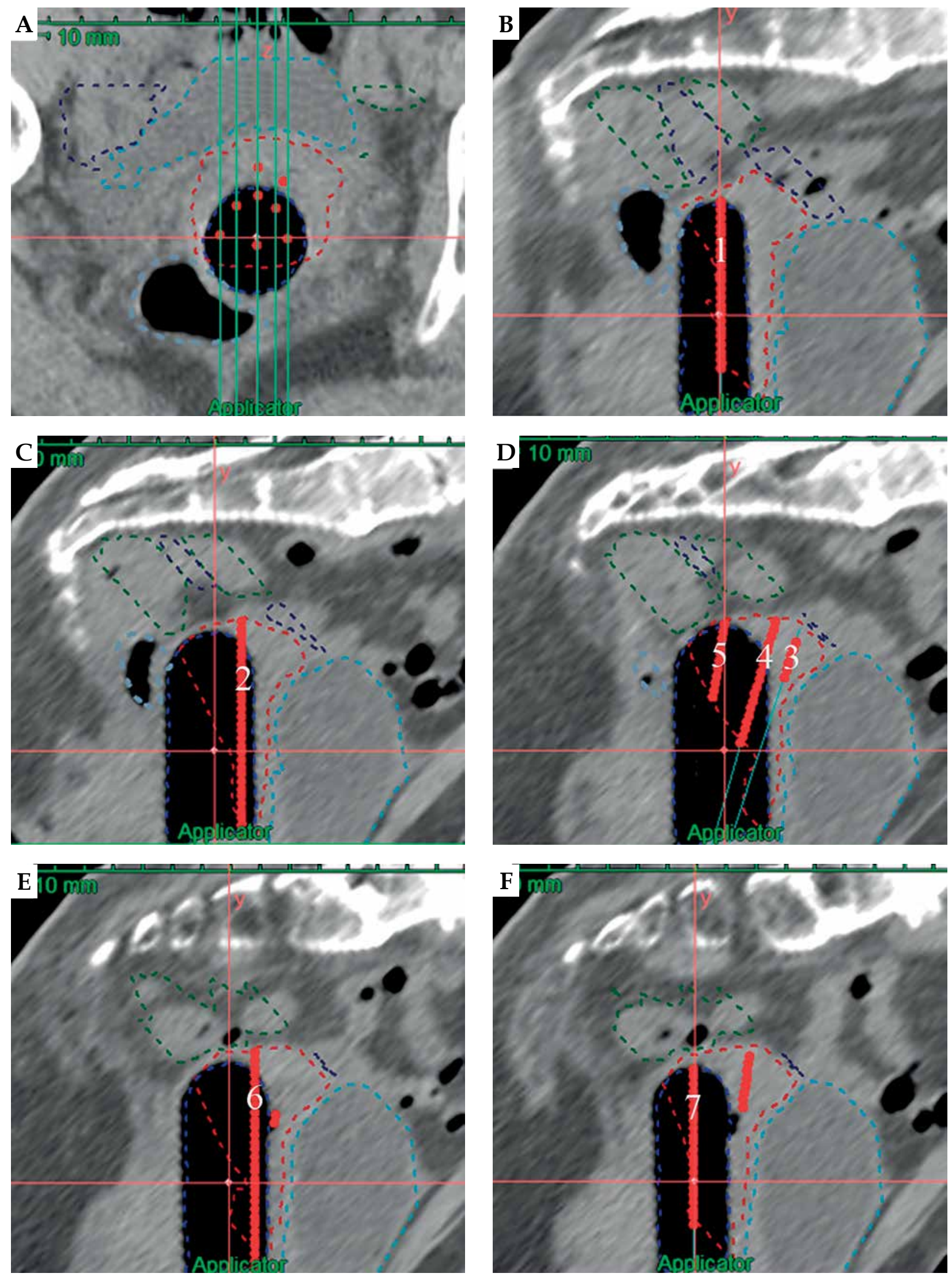

Fig. 3. I. Preplanning: The needle directions parallel to the axis of the applicator: A) the transverse section with labels for the other subgraphs: B) the first, C) the second, D) the third to the fifth, E) the sixth, and F) the seventh needles 

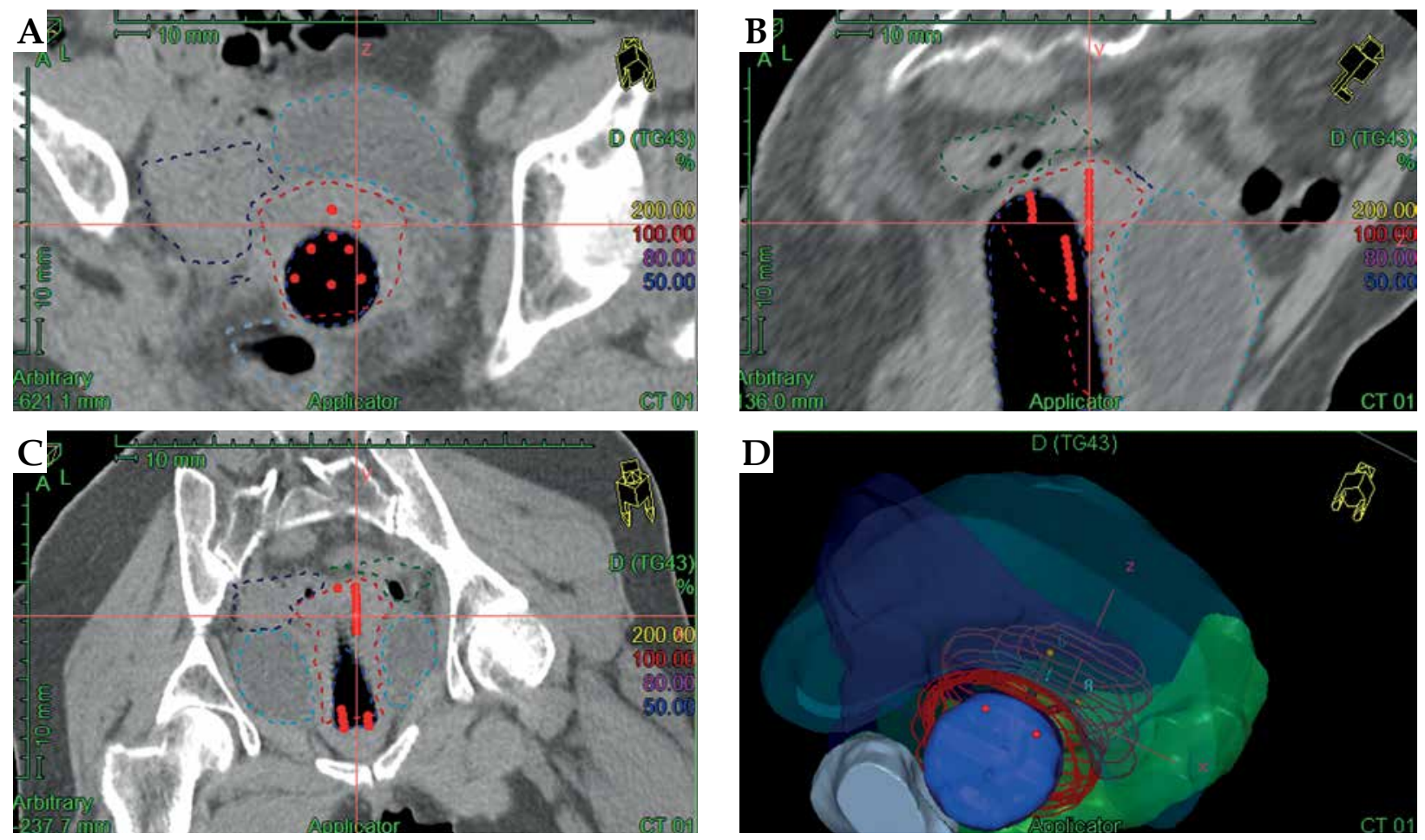

Fig. 4. I. Preplanning: To obtain better target coverage, we set an oblique needle, which was both forward and left with respect to the patient: A) para-axial plane (according to needle No. 8), B) para-sagittal plane (according to needle No. 8), C) para-coronal plane (according to needle No. 8), D) 3D view
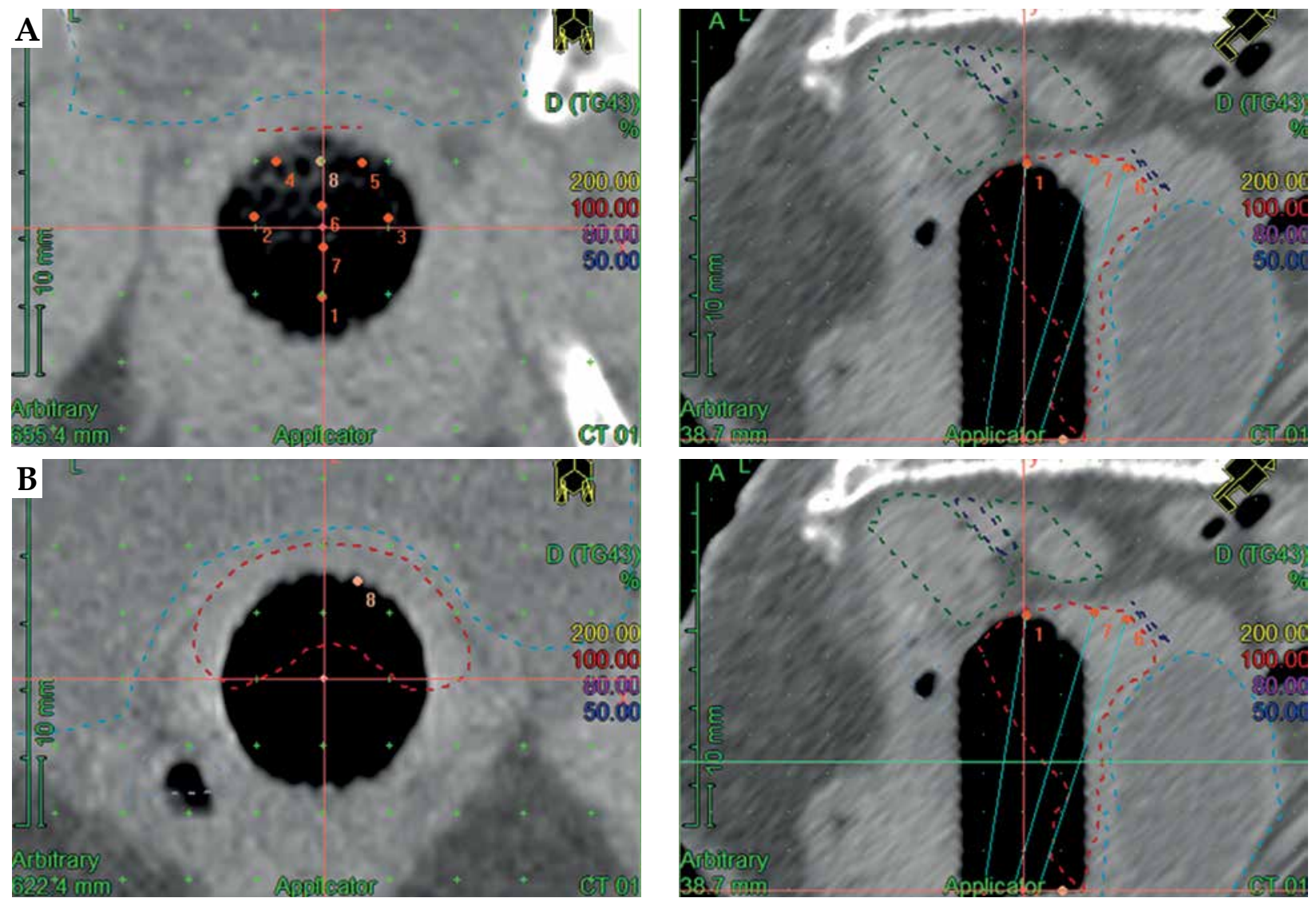

Fig. 5. I. Preplanning: We obtained the directional data of the needles, which are shown in Table 1, according to the relationship between the needles and the coordinate system: A) base end of applicator, B) $3.3 \mathrm{~cm}$ upper from the base end of applicator 

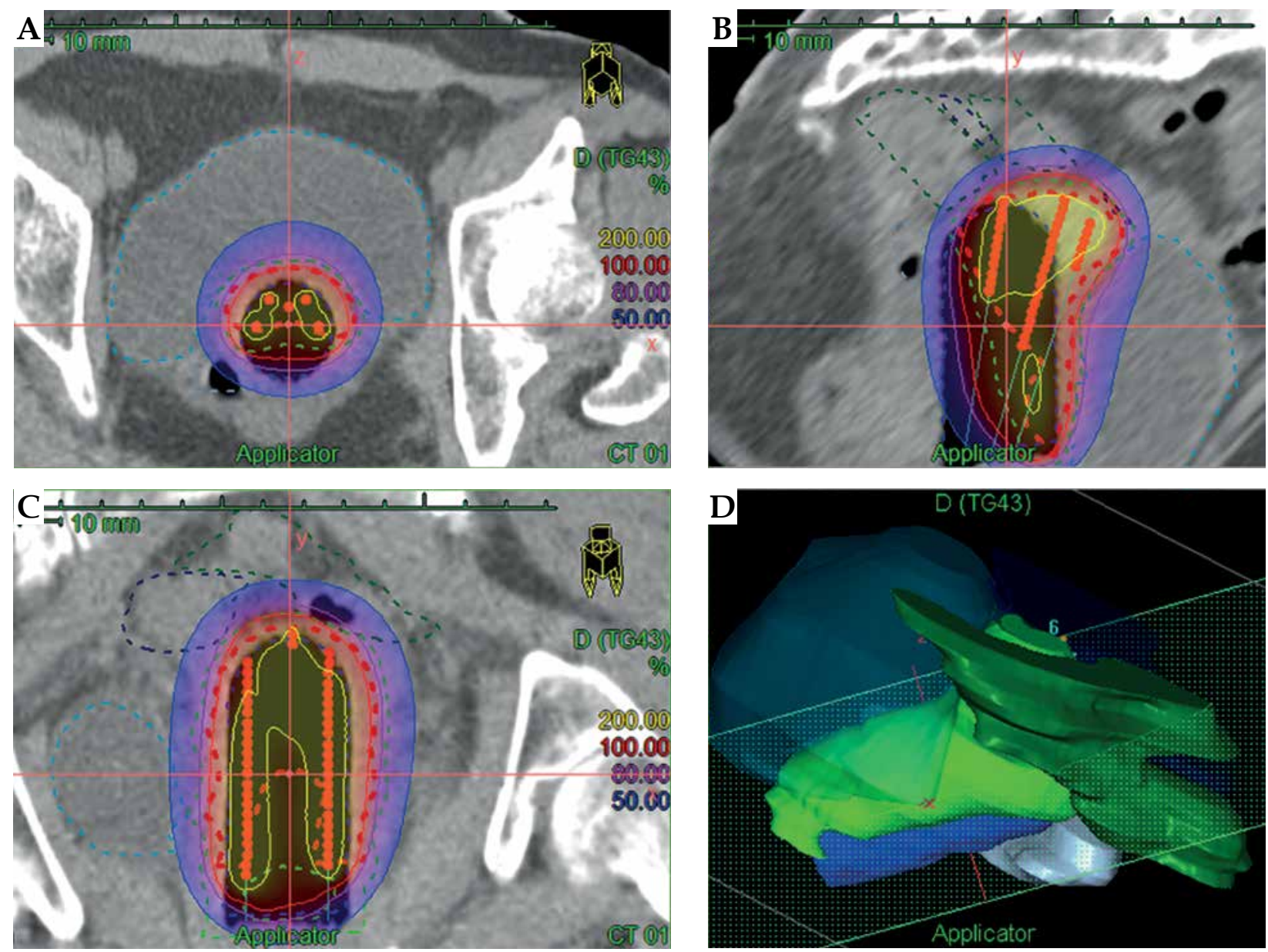

Fig. 6. I. Preplanning: The relationship between the preplanned isodose curves and the target volume: A) para-axial plane (according to applicator), B) sagittal plane, C) para-coronal plane (according to applicator), D) 3D view

applicator is shown in Figures 11-13. The implantation of the applicator and the needles was performed under the guidance of transrectal ultrasound in every application. We used the following brachytherapy treatment schema: 4 fractions, 7 Gy/fraction; 1 week apart for total treatment time of 57 days (including EBRT).

\section{Results}

An example (the first fraction) of the relationship between the isodose curves and the target volumes or organs at risk (OARs) is shown in Figure 14. The DVH parameters for all the treatment plans, including the preplanning phase, are shown in Table 2 . Brachytherapy was well tolerated and achieved a satisfactory total equivalent dose in $2 \mathrm{~Gy} /$ fraction $\left(\mathrm{EQD}_{2}\right)$ for HR-CTV. At the last follow-up at 24 months after brachytherapy, there was no evidence of recurrence in the pelvic region and no signs or symptoms of complications (see Figure 15).

\section{Discussion}

In conventional 2D brachytherapy, individualized treatment cannot be achieved. The use of 3D brachytherapy shows a trend towards increased local control and improved overall survival with reduced toxicity compared
Table 1. The coordinate data for two points of each implant needle $(\mathrm{cm})$

\begin{tabular}{lcccccc} 
Needle & \multicolumn{3}{c}{ Base end } & \multicolumn{3}{c}{ Tip end } \\
\cline { 2 - 7 } number & $x$ & $y$ & $z$ & $x$ & $y$ & $z$ \\
\hline 1 & -1.0 & 0.0 & 0.1 & -1.0 & 6.0 & 0.1 \\
\hline 2 & -0.6 & 0.0 & 1.0 & -0.6 & 6.0 & 1.0 \\
\hline 3 & 0.0 & 0.0 & 0.3 & 0.0 & 7.0 & 2.7 \\
\hline 4 & 0.0 & 0.0 & -0.3 & 0.0 & 7.0 & 1.8 \\
\hline 5 & 0.0 & 0.0 & -1.0 & 0.0 & 7.0 & 0.1 \\
\hline 6 & 0.6 & 0.0 & 1.0 & 0.6 & 6.0 & 1.0 \\
\hline 7 & 1.0 & 0.0 & 0.1 & 1.0 & 6.0 & 0.1 \\
\hline 8 & 0.0 & 0.0 & 1.0 & 0.5 & 3.3 & 1.5
\end{tabular}

to 2D brachytherapy [12]. At present, individualized treatment is a recommended strategy for cervical cancer brachytherapy in the modern management of cervical cancer $[8,13]$. This paper introduced the design and application of an individualized cylindrical vaginal applicator using 3D modeling and printing technologies and 


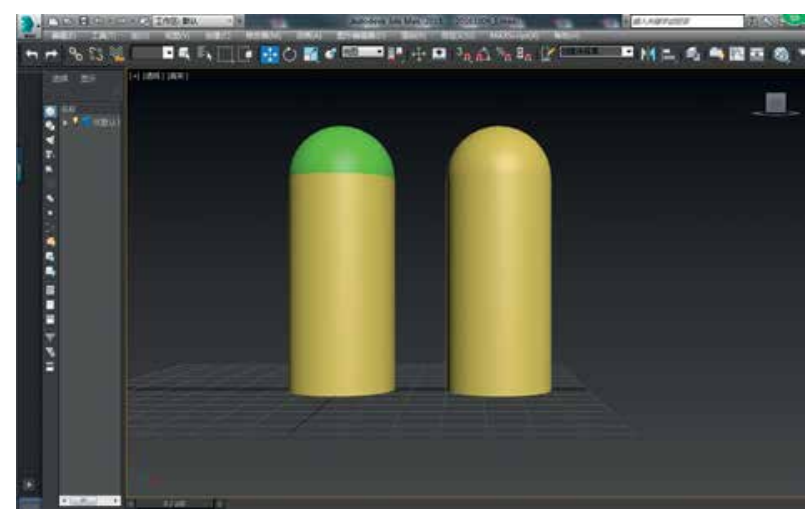

Fig. 7. II. 3D modeling: Modeling of the main body of the vaginal applicator without guide holes: the Boolean union of a cylinder and a sphere

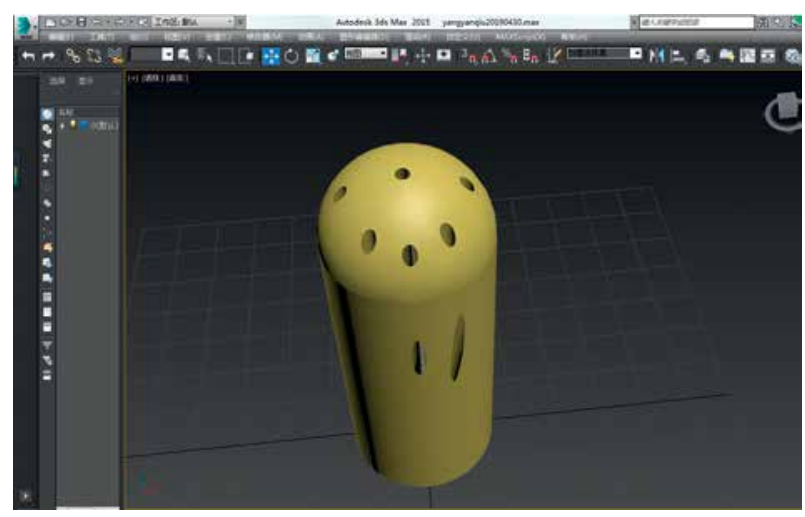

Fig. 9. II. 3D modeling: A cylindrical applicator with oblique guide holes was obtained using Boolean subtraction

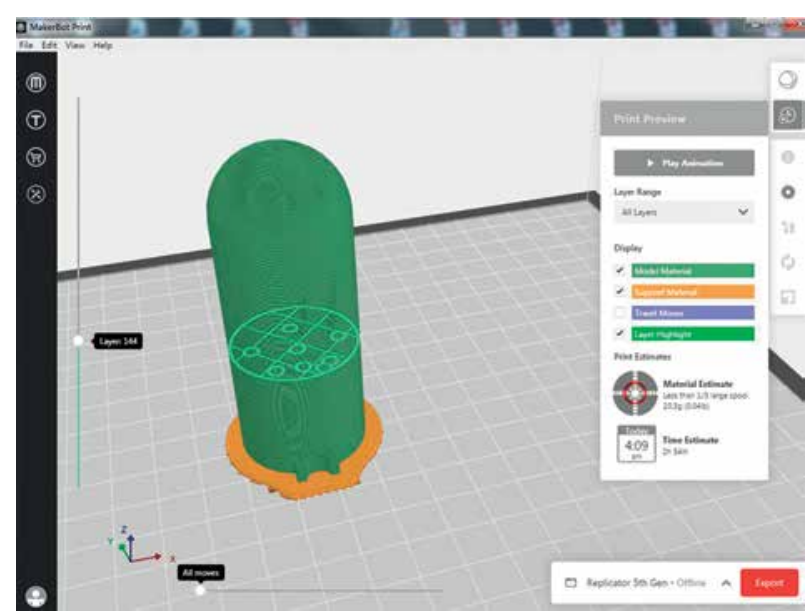

Fig. 11. III. 3D printing: The modeling data were exported as an "*.stl" file to MakerBot Print (version 3.0, MakerBot Industries, USA). In MakerBot Print, the file was processed and exported as a "*. makerbot" file to the 3D printer

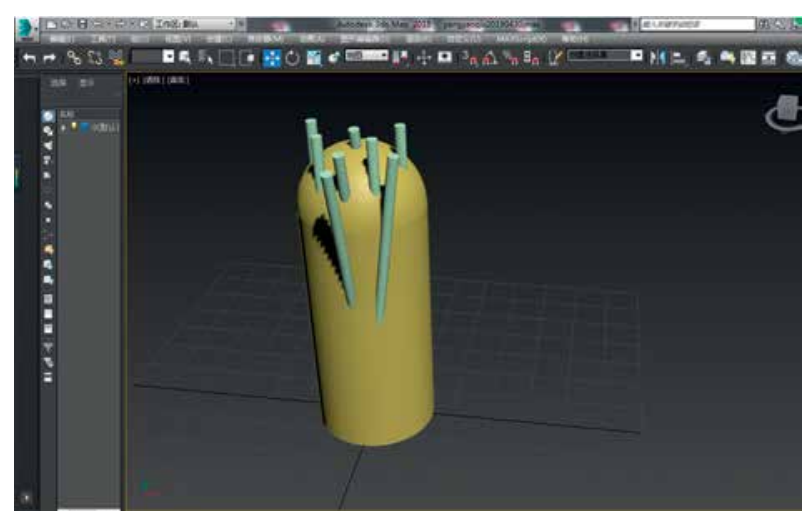

Fig. 8. II. 3D modeling: The needles were constructed as cylinders (shown within the main body of the vaginal applicator), and the distribution and direction were adjusted according to the data shown in Table 1

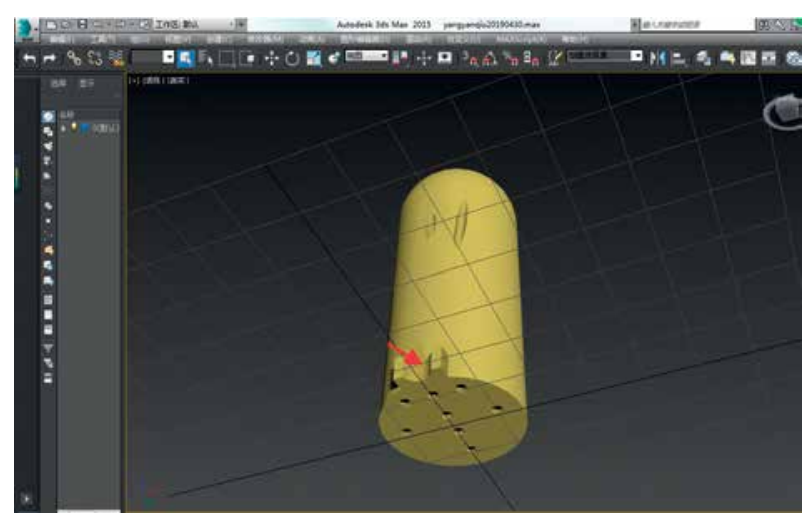

Fig. 10. II. 3D modeling: A mark (red arrow) was included on the main body of the applicator, which just pointed to the anterior of the patient; the Foley catheter passed through this mark

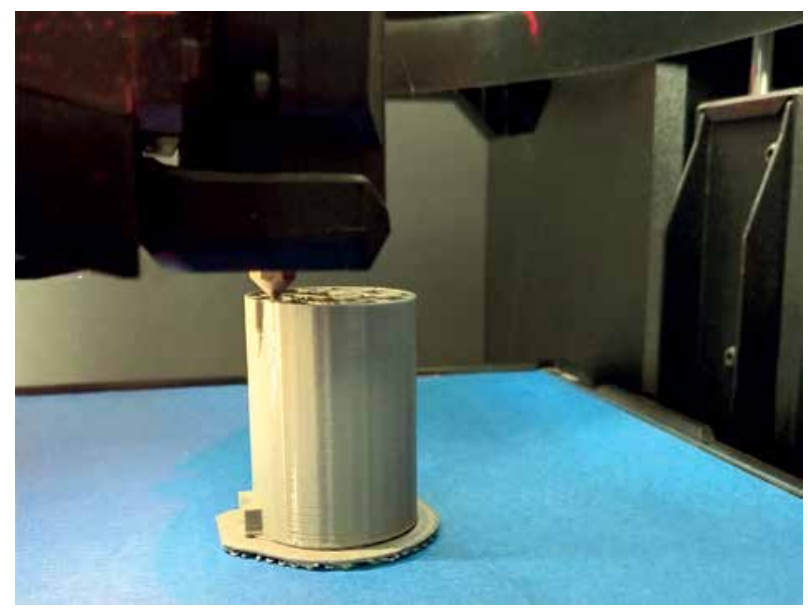

Fig. 12. III. 3D printing: The model was printed using a 3D printer (Replicator + , MakerBot USA) with polylactic acid (PLA) material 

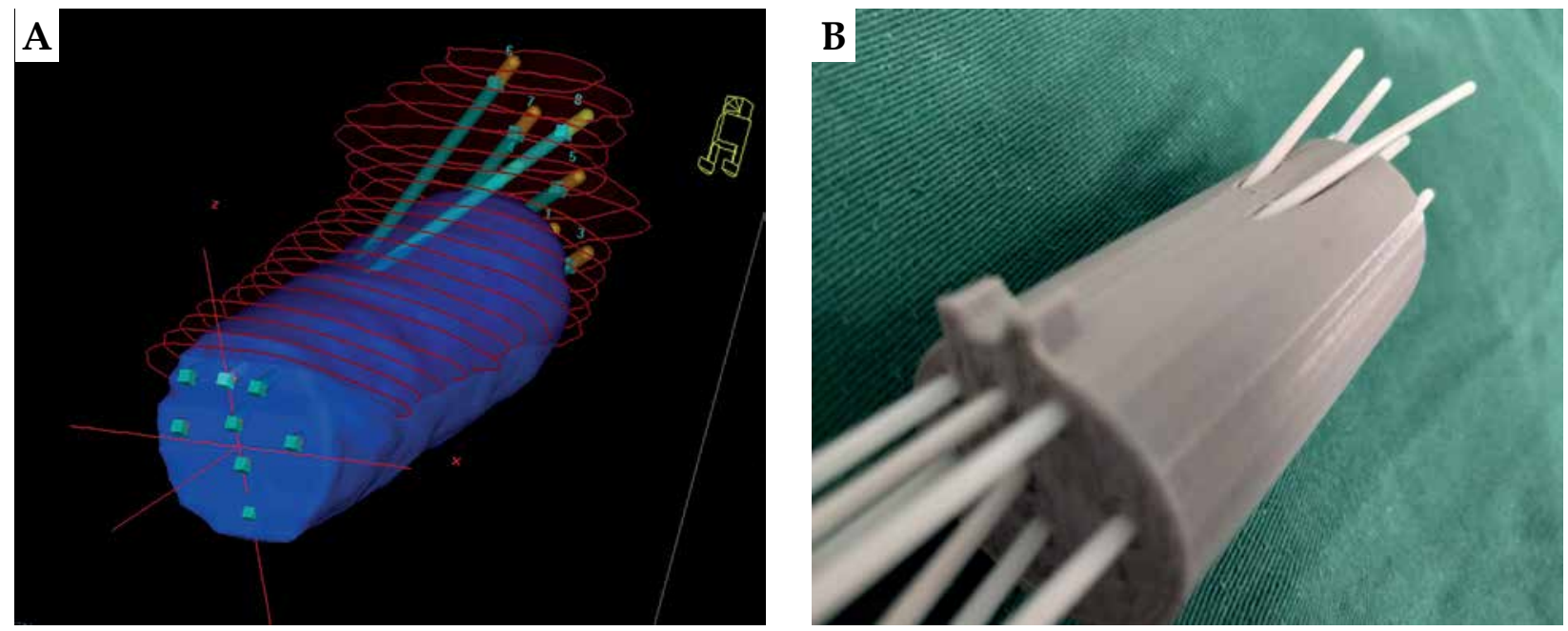

Fig. 13. III. 3D printing: The 3D graphics in the preplanning phase (A) and the cylindrical applicator with oblique needles after 3D printing (B) were compared
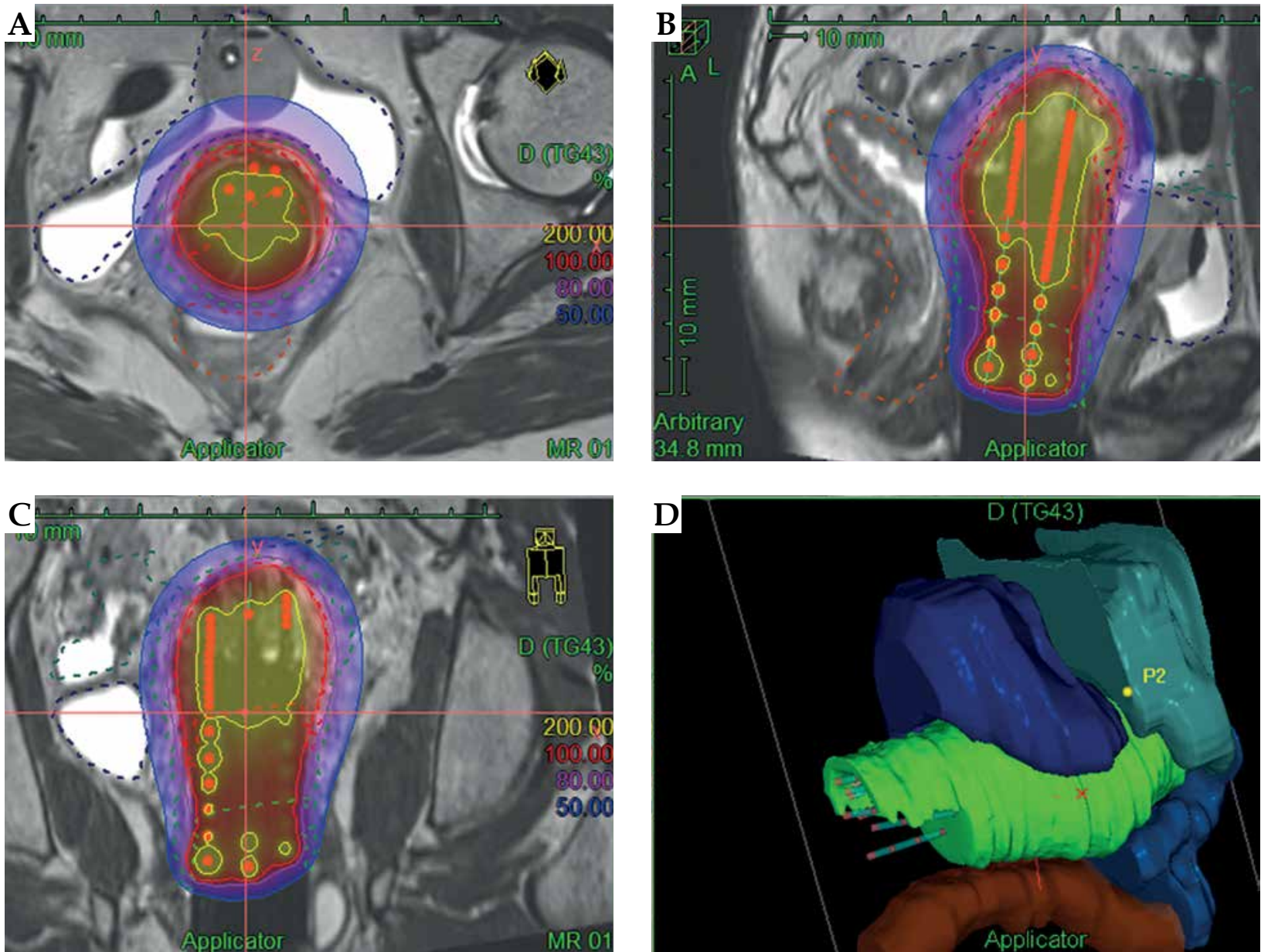

Fig. 14. IV. Treatment plan: An example (the first fraction) of the relationship between the isodose curves and the target volumes or OARs: A) para-axial plane (according to applicator), B) sagittal plane, C) para-coronal plane (according to applicator), D) 3D view 
Table 2. Dose parameters of target volumes and OARs (cGy)

\begin{tabular}{lcccccccc} 
Parameter & Preplanning & BT1 & BT2 & BT3 & BT4 & Total $^{*}$ & Planning aims $^{* *}$ & Limits dose $^{* *}$ \\
\hline HR-CTV $D_{98}$ & 588 & 618 & 660 & 548 & 591 & 7662 & $>7500$ & - \\
\hline HR-CTV $D_{90}$ & 714 & 724 & 756 & 732 & 732 & 8684 & $>9000$ & $>8500$ \\
\hline HR-CTV $D_{50}$ & 1177 & 1157 & 1112 & 1200 & 1173 & 12786 & - & - \\
\hline IR-CTV $D_{98}$ & 415 & 415 & 501 & 318 & 387 & 6338 & $>6000$ & - \\
\hline Bladder $D_{0.1 c c}$ & 659 & 691 & 638 & 570 & 539 & 8783 & - & $<$ \\
\hline Bladder $D_{2 c c}$ & 571 & 531 & 550 & 474 & 443 & 7530 & $<8000$ & - \\
\hline Rectum $D_{0.1 c c}$ & 493 & 445 & 544 & 542 & 383 & 7337 & - & $<9000$ \\
\hline Rectum $D_{2 c c}$ & 383 & 361 & 403 & 381 & 294 & 6232 & $<6500$ & $<7500$ \\
\hline Sigmoid $D_{0.1 c c}$ & 677 & 633 & 392 & 617 & 557 & 8130 & - & $<7500$ \\
\hline Sigmoid $D_{2 c c}$ & 490 & 467 & 292 & 436 & 417 & 6622 & $<7000$ & $<7500$
\end{tabular}

${ }^{*}$ Total dose was $E Q D_{2}$, which is the dose using a linear-quadratic $(L Q)$ equation with $\alpha / \beta=10$ for a tumor and $\alpha / \beta=3$ for OARs, including 45 Gy $/ 25 f E B R T$. ${ }^{*}$ Objective recommendation from EMBRACE II
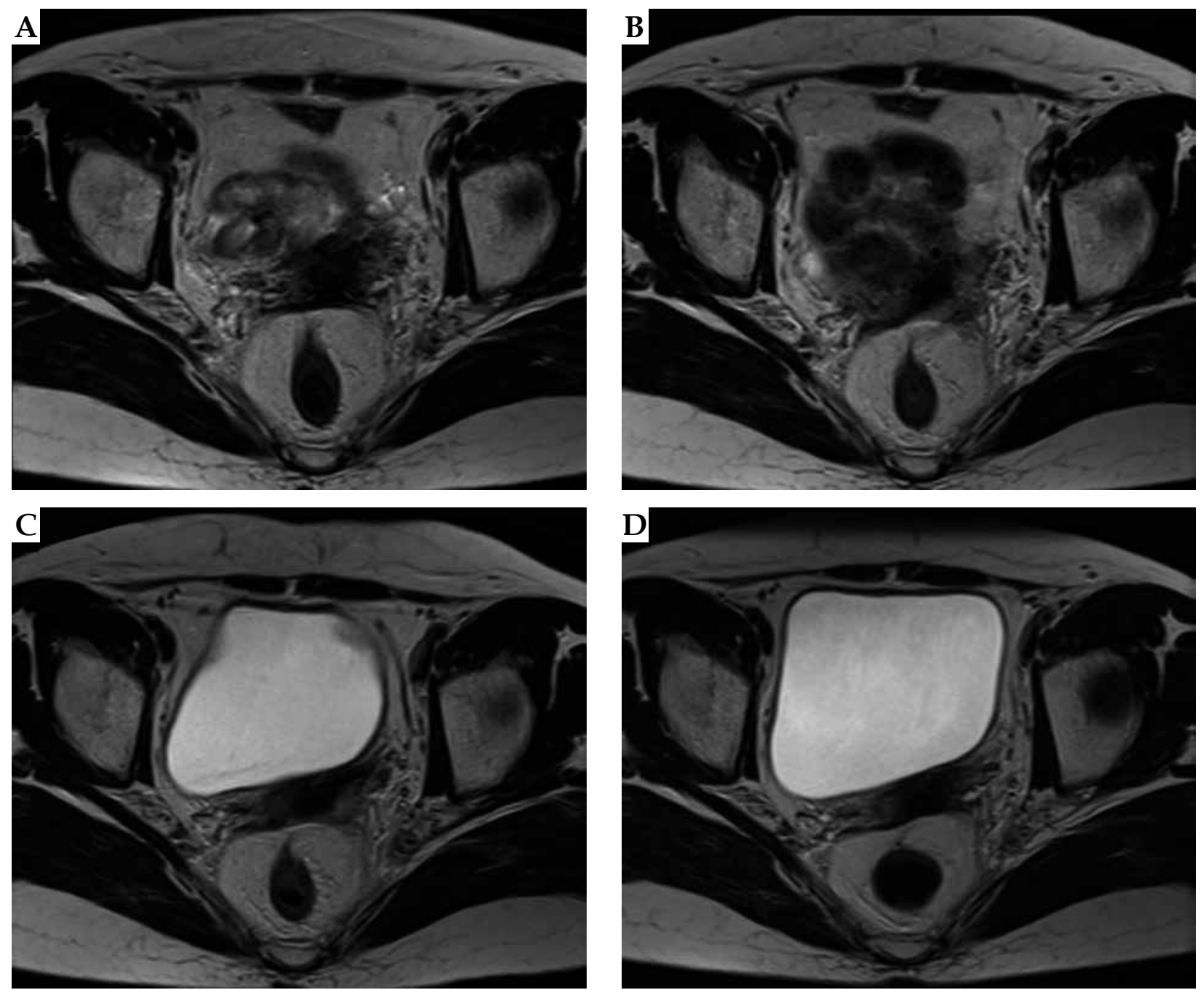

Fig. 15. IV. Clinical outcome: MRI showed no evidence of recurrence in the pelvic region at A) 9 months, B) 12 months, C) 16 months, and D) 24 months after brachytherapy 
achieved good clinical results. This paper introduces only the production process of the individualized vaginal cylindrical applicators. The specific 3D modeling software and 3D printer used in this study are replaceable. As long as other software and hardware have the corresponding functions, they can be used to replace the software and hardware introduced in this paper. In the preplanning phase, the delineation of target volumes and OARs is not directly based on MRI but on CT images cross-referenced with an MRI image. Although this method is not the best strategy, it is an acceptable secondary option to some extent. Readers can follow the workflow of this study and perform the preplanning phase based on MRI, which will bring more optimized results. In this study, the design of an individualized cylindrical vaginal applicator was based on the data acquired in the preplanning phase, which is an effective method, and preplanning is also recommended in all brachytherapy plans [14].

Four fractions of brachytherapy achieved the ideal dose distribution. Figure 6 shows that the isodose line of the prescribed dose covered the HR-CTV well and avoided OARs well. From Table 2, it can be seen that the total $\mathrm{EQD}_{2}$ of the target volumes and the OARs all achieved the limit dose, and 5 of the 7 items achieved the planning aim dose. This result fully shows that the individualized cylindrical vaginal applicator using 3D modeling and printing technologies has good availability, practicability and repeatability.

For cervical cancer brachytherapy after subtotal hysterectomy, cylindrical vaginal applicators are commonly used applicators. Because of the high dose gradient in brachytherapy, when the depth of vaginal infiltration is far greater than $5 \mathrm{~mm}$, it is impossible to achieve both a high dose coverage of the prescribed dose for the target volumes and a low dose to OARs [6]. The individualized applicator introduced in this paper can be used for deep vaginal invasion, vaginal tumors with asymmetric growth, vaginal recurrences, and vaginal cuff recurrence after subtotal hysterectomy.

\section{Conclusions}

When standardized applicators cannot achieve a good dose distribution for cervical cancer after subtotal hysterectomy, one can achieve a higher dose coverage of the target and better sparing of the OARs by using a 3D-printed, individualized cylindrical vaginal applicator.

\section{Disclosure}

The authors report no conflict of interest.

\section{References}

1. Dimopoulos JC, Pötter R, Lang S et al. Dose-effect relationship for local control of cervical cancer by magnetic resonance image-guided brachytherapy. Radiother Oncol 2009; 93: 311-315.

2. Mazeron R, Castelnau-Marchand P, Dumas I et al. Impact of treatment time and dose escalation on local control in locally advanced cervical cancer treated by chemoradiation and image-guided pulsed-dose rate adaptive brachytherapy. Radiother Oncol 2015; 114: 257-263.
3. Kirisits C, Lang S, Dimopoulos J et al. The Vienna applicator for combined intracavitary and interstitial brachytherapy of cervical cancer: Design, application, treatment planning, and dosimetric results. Int J Radiat Oncol Biol Phys 2006; 65: 624-630.

4. Nomden CN, de Leeuw AA, Moerland MA et al. Clinical use of the Utrecht applicator for combined intracavitary/interstitial brachytherapy treatment in locally advanced cervical cancer. Int J Radiat Oncol Biol Phys 2012; 82: 1424-1430.

5. Oike T, Ohno T, Noda SE et al. Can combined intracavitary/interstitial approach be an alternative to interstitial brachytherapy with the Martinez Universal Perineal Interstitial Template (MUPIT) in computed tomography-guided adaptive brachytherapy for bulky and/or irregularly shaped gynecological tumors? Radiat Oncol 2014; 9: 222.

6. Gebhardt BJ, Vargo JA, Kim H et al. Image-based multichannel vaginal cylinder brachytherapy for the definitive treatment of gynecologic malignancies in the vagina. Gynecol Oncol 2018; 150: 293-299.

7. Sethi R, Cunha A, Mellis K et al. Clinical applications of custom-made vaginal cylinders constructed using three-dimensional printing technology. J Contemp Brachytherapy 2016; 8: 208-214.

8. Lindegaard JC, Madsen ML, Traberg A et al. Individualised 3D printed vaginal template for MRI guided brachytherapy in locally advanced cervical cancer. Radiother Oncol 2016; 118: 173-175.

9. Albano M, Dumas I, Haie-Meder C. Brachytherapy at the Institut Gustave-Roussy: personalized vaginal mould applicator: technical modification and improvement. Cancer Radiother 2008; 12: 822-826.

10. Wiebe E, Easton H, Thomas G et al. Customized vaginal vault brachytherapy with computed tomography imaging-derived applicator prototyping. Brachytherapy 2015; 14: 380-384.

11. Haie-Meder C, Potter R, Van Limbergen $E$ et al. Recommendations from Gynaecological (GYN) GEC-ESTRO Working Group (I): concepts and terms in 3D image based 3D treatment planning in cervix cancer brachytherapy with emphasis on MRI assessment of GTV and CTV. Radiother Oncol 2005; 74: 235-245.

12. Derks K, Steenhuijsen JLG, van den Berg HA et al. Impact of brachytherapy technique (2D versus 3D) on outcome following radiotherapy of cervical cancer. J Contemp Brachytherapy 2018; 10: 17-25.

13. Foroudi F, Bull CA, Gebski V. Radiation therapy for cervix carcinoma: benefits of individualized dosimetry. Clin Oncol (R Coll Radiol) 2002; 14: 43-49.

14. Chakrabarti B, Pal SK, Sepai HM et al. Clinical and dosimetric consequences of imperfect applicator insertion in cervical cancer brachytherapy. J Contemp Brachytherapy 2018; 10: 321336. 
